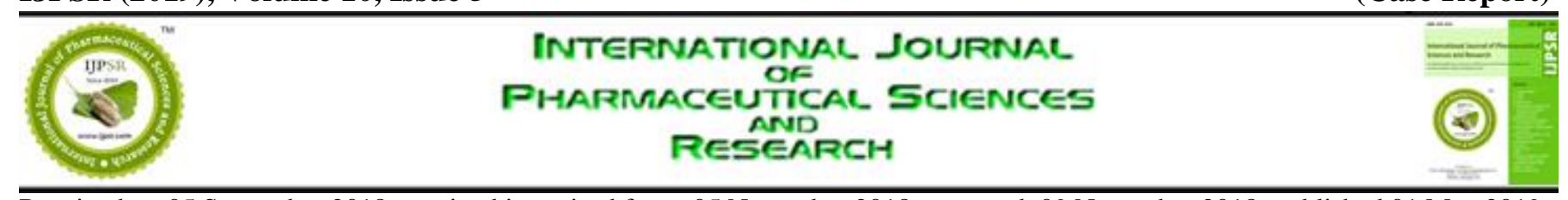

Received on 05 September 2018; received in revised form, 05 November 2018; accepted, 09 November 2018 ; published 01 May 2019

\title{
XANTHOGRANULOMATOUS PYELONEPHRITIS- A RARE CASE REPORT
}

\author{
K. Sai Vasudev ${ }^{*}{ }^{1}$, T. Avinash ${ }^{1}$ and R. Hari Babu ${ }^{2}$
}

Department of Pharmacy Practice ${ }^{2}$, Chebrolu Hanumaiah Institute of Pharmaceutical Sciences ${ }^{1}$, Guntur 522503, Andhra Pradesh, India.

Keywords:

Xanthogranulomatous pyelonephritis, Nephrectomy, E. coli, Haematuria

Correspondence to Author:

K. Sai Vasudev

VI/VI Pharm. D,

Chebrolu Hanumaiah Institute of

Pharmaceutical Sciences, Guntur 522503, Andhra Pradesh, India.

E-mail: konasaivasudev@gmail.com

\begin{abstract}
An unusual type of the long-lasting pyelonephritis demonstrating a long-lasting granulomatous disease resulting in a nonfunctional kidney in the patient is generally linked with xanthogranulomatous pyelonephritis. The name itself suggests this involves a granulomatous progression that is chronic, due to a chronic or sub-acute infection in the patient inciting a chronic but incomplete immune reaction in the individual. Different bacteria are generally known to be identified, but the most commonly associated species are Escherichia coli and Proteus mirabilis. Clinical presentation of the patient suffering from the disease is typically unclear, consisting of symptoms like malaise, loss of weight and low-grade fever and loss of appetite. Blood in the urine (hematuria) and flank pain are generally presented in the patients with the disease. Urinary tract symptoms may not be present always, but pus in the urine and positive urinary cultures are present in many of the cases of the patients (95 and 60\% respectively). The disease finally results in the replacement of the kidney by a mass of reactive tissue, provoking staghorn calculus with associated hydronephrosis to any of the gradations. Foamy macrophages that are lipid-laden generally prevail. The inflammatory process eventually extends into perinephric tissues and even adjacent organs of the body. MRI, CT scan, ultrasound examination helps in diagnosing the disease. Management includes partial or complete nephrectomy and antibiotics. In this case report, we will include a case of severe xanthogranulomatous pyelonephritis managed with nephrectomy.
\end{abstract}

INTRODUCTION: This disease was for the first time mentioned and elucidated by Schlagenhaufer in the year of 1916. This disease is a long-lasting inflammatory, rare, serious disorder affecting the organ kidney characterized by a destructive mass invading the renal parenchyma.

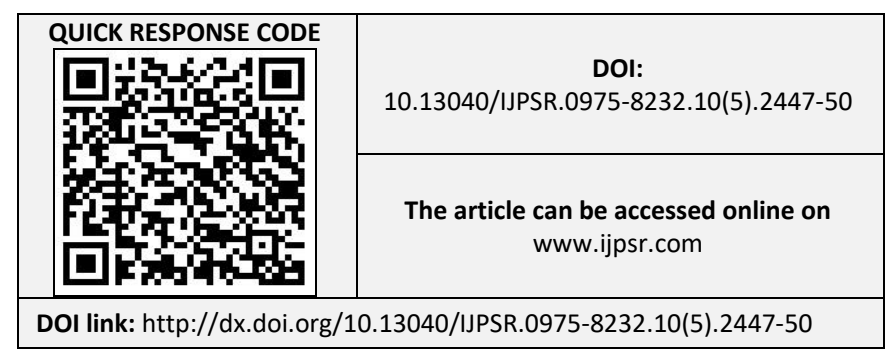

The precise etiology of this disease was not identified. The disease needs long-term obstruction of the kidney along with the infection and insufficient patient response to that acute inflammatory response to develop into XGP.

Bacteria like Proteus, E. coli, and Pseudomonas are the most commonly identified organisms in the urine culture of the patients. Nearly in $75-85 \%$ of the patient's staghorn calculi develop. Diabetes mellitus, the immuno-compromised status may also be associated with the condition. Abnormal lipid metabolism in the patient may also be an etiological factor for the disease. 
This disease shares a tumor like properties like invasion and damage of the focal tissue. Surrounding organs of the kidney such as lower gastrointestinal tract organs can also be involved and affected by the disease ${ }^{1}$. Coming to the presentation of the patient they look ill chronically and with loss of appetite, raised body temperature associated with chills, weight loss, and flank pain. Formation of the fistulas may be seen in some patients. Obstructive calculi are the common presentation in pediatric patients. Post-operative sepsis from the disease has a greater mortality rate. This disease is related to carcinoma (transitional cell) of the renal pelvis. Consider nephrectomy as the treatment option in a diffuse or advanced stage of the disease.

Antibiotics can be an option in case of focal or segmental disease if diagnosed in the early stage preoperatively. Broad-spectrum antibiotics and symptomatic management are used both before and after the surgery which is the important aspect in the successful management and for good prognosis of the patient. Nephrectomy is a complex procedure but definitive management for XGP ${ }^{2}$. Chief complications may include infection, postoperative pneumonia, hemorrhage, and death ${ }^{3}$. We present a case of XGPN managed with nephrectomy of the left kidney. No postoperative complications occurred in our patient.

CASE REPORT: A 8-year-old boy was presented to the Urology Clinic with the complaints of on and off type fever since 3 months, puffiness of face and abdomen since 1 month and left sided abdominal pain and decreased urinary passage since 10 days that developed before the presentation. The patient had a similar past medical history 2 months before the present complaints. He is immunized according to the NIH, and the patient had no developmental delay. He is a student. His family history was not significant for any renal or urologic illness. Abnormal lab data includes $\mathrm{Hb}-10 \mathrm{~g} \%$, platelets7.9 lakhs/microliter (Reactive thrombocytosis), ESR- $70 \mathrm{~mm} / \mathrm{h}$, WBC- 15,100 / microliter. CT scan of the abdomen revealed the presence of multiple small two mm calculi of size $(2.4 \times 1.4) \mathrm{cm}$ were noted in the renal pelvis extending into lower pole calyx, and moderate hydronephrosis is present. Mild fat stranding is noted around the renal pelvis and bulk sized left kidney. The right kidney was normal sized with no calculi and no hydronephrosis. Urine sample culture showed the presence of $E$. coli after twenty-four-hour culture. Urinalysis revealed the presence of plenty of epithelial cells and pus cells.

Based on these clinical manifestations and laboratory results he was diagnosed with xanthogranulomatous pyelonephritis with renal calculi and was prescribed with the following medications.

- Tab Ceftum (Cefuroxime)- 250 mg BD PO from hospital day 1-11.

- Tab Paracetamol 500 mg BD PO from day 1-4.

- Syp Corex (Codiene phosphate $4 \mathrm{mg} / 5 \mathrm{ml}$, Chlorpheniramine maleate $10 \mathrm{mg} / 5 \mathrm{ml}$ ) - $5 \mathrm{ml}$ BD PO from day 4-11.

- Inj Pantop (pantoprazole) - 20 mg IV OD from day $1-11$.

- Inj Monocef (Ceftriaoxone)- 1g IV BD on day 4 (preoperatively), 8 (preoperatively), 10 (preoperatively).

A ureteral stent was placed on day 4, PCNLpercutaneous nephrolithotomy was performed on day 8 , and left kidney nephrectomy was done on day 10 .

The patient was counseled regarding the health benefits. The patient was prescribed with Cefuroxime $250 \mathrm{mg}$ orally twice daily, Paracetamol $500 \mathrm{mg}$ orally twice daily for treating fever, PCNL was planned for management of renal calculi and nephrectomy of the left kidney was carried out for definitive management of XGP. The patient did not develop any postoperative complications and was discharged. The patient and the caretakers were instructed about the care that is to be taken in nephrectomy patients and the medications that are contraindicated and the medications that are to be used with caution.

DISCUSSION: Patients suffering from the disease will have pain in the abdomen, increased body temperature, loss of appetite, weight loss, blood in urine, difficulty in urination, infections of the urinary tract that are resistant to various antibiotics, or a mass in the abdomen that is palpable ${ }^{4}$ and here this patient also had the complaints of fever, dysuria. 
The disease was classified into three stages by Malek and Elder: Stage I where nephric disease confined to the kidney but doesn't involve the other organs; Stage II, extending into the gerota fascia of the kidney; and Stage III, disease extending into adjacent perinephric space and other retroperitoneal structures apart from the kidney alone and here this patient belongs to stage II of the grading. It has been described as a great imitator of other diseases, because of its varied clinical presentations; it is most generally misdiagnosed as a renal mass ${ }^{5,6,7}$.

By using ultrasonography, we can differentiate between the two forms of the disease: a diffuse form of the disease (83\%-90\%) and focal form of the disease $(10 \%-17 \%)$. In the diffuse form of the disease, there will be a diffuse enlargement of the kidney with multiple hypoechoic areas and hyperechoic foci. Hypoechoic areas indicate the calyceal or pelvicalyceal dilatation and parenchymal destruction. Hyperechoic foci with posterior acoustic shadowing indicate the renal calculi and debris in hydronephrosis. The disease confines to only one part or one pole of the kidney in the focal form of the disease ${ }^{8}$. The computer tomography scan is considered the diagnostic modality of choice for this rare disease and the patients CT scan indicated the presence of renal calculi and moderate hydronephrosis.

The characteristic features in diffuse form of the disease are generalized renal enlargement, perinephric fat stranding, and thickening of the gerota fascia but they are not pathognomonic. Here the patient presented with bulk sided left kidney and fat stranding around the renal pelvis. We can observe a distinguishing sign called the "bear paw sign" in the diffuse form of the disease, and this sign refers to the cross-sectional appearance of the kidney in the patient suffering from the disease, which looks like the paw of a bear. We can observe the contraction of the renal pelvis although the calyces of the kidney are dilated, that resembles the toe pads of the paw. In focal disease, the CT scan shows focal mass lesion, occupying one pole or an enhancing low-density mass lesion, surrounding dilated, stone-filled calyces ${ }^{9}$.

The most common causative organisms are Proteus mirabilis and Escherichia coli, although sterile urine is found in one-third of the cases. The patient in this case report has a positive urine sample cultured with $E$. coli indicating the causative organism. Care should be taken to include this disease to differentiate them from other diseases of the pediatrics presenting with psoas abscess, perirenal renal mass and non-functioning kidney with / or without urolithiasis. There should be high awareness clinically, and high levels of suspicion are required to achieve the accurate pre-surgical diagnosis and appropriate management of the disease. In this patient, XGP is chiefly attributed to the long stranding renal calculi and infection. Elevated ESR and elevated WBC count are also attributed to the infection. Left abdominal pain is chiefly due to renal calculi.

Treatment of the disease with medical management alone is incomplete without surgery. Antibiotics only provide a temporary measure for patients that require medical work-up before nephrectomy. The gold standard of treatment for the disease is Total nephrectomy (Removal of the kidney) unless both sides are affected in which case partial nephrectomy is preferred. The prognosis of the patient is considered to be good after the management of the disease.

CONCLUSION: This case report delivers the highlights needed to familiarize the physicians about this rare, life threating, uncommon entity. It should be differentially diagnosed with renal tuberculosis, renal abscess, renal cell carcinoma (RCC), angiomyolipoma (AML). XGP if left untreated leads to RCC and death

CONSENT: Written informed consent was obtained from the patient for publication of this case.

FUNDING: No funding sources

ACKNOWLEDGEMENT: I am thankful to my Lecturers and Professors of Chebrolu Hanumaiah Institute of Pharmaceutical Sciences.

CONFLICT OF INTEREST: There is no conflict of interest.

\section{REFERENCES:}

1. Gali V, Hemalatha G and Suresh N: An interesting case report- Xanthogranulomatous pyelonephritis masquerading as a tumor. Sch J App Med Sci 2016; 4(9D): 3454-56. 
2. Goyal S, Gupta M and Goyal R: Xanthogranulomatous pyelonephritis: A rare entity. N Am J Med Sci 2011; 3(5): 249-50.

3. Bingol-Kologlu M, Ciftci AO, Senocak ME, Tanyel FC, Karnak I and Buyukpamukcu N: Xanthogranulomatous pyelonephritis in children: Diagnostic and therapeutic aspects. Eur J Pediatr Surg 2002; 12(1): 42-8.

4. Marinacci LX and Rosales I: A case report on Xanthogranulomatous pyelonephritis. NEJM 2018; 378: 40.

5. Craig WD, Wagner BJ and Travis MD: Pyelonephritis: Radiologic-pathologic review. Radio Graphics 2008; 28(1): 255-76.

6. Jae-Hyuk Y: Delayed diagnosis of xanthogranulomatous pyelonephritis in a quadriplegic patient with uncontrolled cutaneous fistula: A case report. Medicine 2018; 97(2): e9659.

7. Buttice S, Inferrera A and Ascenti G: Xanthogranulomatous pyelonephritis can simulate a complex cyst: Case description and review of the literature. Urology Case Reports 2014; 2(3): 113-15.

8. Friedl A, Tuerk C, Schima W and Broessner C: Xanthogranulomatous pyelonephritis with staghorn calculus, acute gangrenous appendicitis and enterocolitis: A multidisciplinary challenge of kidney-preserving conservative therapy. Curr Urol 2014; 8: 162-65.

9. Jagdale A, Mittal S, Patel K, Azhar S and Prasla S: Xanthogranulomatous pyelonephritis: A case review of two cases. Int J Res Med Sci 2016; 4(9): 4198-01.

How to cite this article:

Vasudev KS, Avinash T and Babu RH: Xanthogranulomatous pyelonephritis- A rare case report. Int J Pharm Sci \& Res 2019; 10(5): 2447-50. doi: 10.13040/IJPSR.0975-8232.10(5).2447-50.

All @ 2013 are reserved by International Journal of Pharmaceutical Sciences and Research. This Journal licensed under a Creative Commons Attribution-NonCommercial-ShareAlike 3.0 Unported License.

This article can be downloaded to Android OS based mobile. Scan QR Code using Code/Bar Scanner from your mobile. (Scanners are available on Google Play store) 